\title{
PROBE EFFICIENCY IMPROVEMENT WITH REMOTE AND TRANSMISSION LINE TUNING AND MATCHING
}

\author{
P. Villa, J.J. Vaquero, $†$ S. Chesnick, + and J. Ruiz-Cabello \\ *Unidad de RMN \& Dept. Química-Física II, Fac. Farmacia, Universidad Complutense, Madrid 28040, Spain; $†$ Nuclear Medicine \\ Department, Clinical Center and \$Laboratory of Cardiac Energetics, In vivo NMR Center; National Institutes of Health, \\ Bethesda, MD 20892-1180, USA
}

\begin{abstract}
In certain nuclear magnetic resonance (NMR) applications in which accessibility to the gantry is limited, performing optimal tuning and matching represents a major problem. Here, we discuss a method of tuning NMR probe circuits and matching their impedances which uses cables with different impedance values. This simple but efficien method may be advantageous compared with much more difficul perfect tuning and matching.
\end{abstract}

Keywords: Matching and tuning network; Probe; Transmission line.

\section{INTRODUCTION}

Precise probe tuning and matching plays an important role in many nuclear magnetic resonance (NMR) experiments. This procedure is not fully automated in every system, and in certain biologic and medical applications access to the tuning and matching elements inside the magnet bore becomes difficult making the process laborious and time consuming.

Different authors suggested the possibility of remotely tuning the probes under such conditions by placing variable tuning capacitors at an even number of quarter-wavelength distances along a transmission line. ${ }^{1-4}$ The relationship between the power transfer from the radio frequency amplifie to the probe during the transmission mode, as well as the power of the signal detected at the probe that reaches the preamplifie during reception (efficienc of the coil) relies in part on the probe matching the characteristic impedance $Z_{0}$ of the transmission line used (usually $50 \Omega$ ). ${ }^{4}$ Since matching is realized after the firs segment of transmission line placed between the input/output electronics and the probe (Fig. 1A), this type of remote tuning carries a cost in efficiency The reduction of power forwarded to the probe itself is caused by the mismatch (and subsequent reflection at the probe/transmission line junction. To reduce this reflectio Rath $^{4}$ proposes to introduce a partial matching circuit to the transmission line impedance (Fig. 1B), technique can increase the efficienc up to $90 \%$, three times higher than the value achieved with the half-wave length matching only. This approach has the disadvantage of having a narrow frequency range, and it has to be tailored to each probe.

In this communication, we present the results achieved with a similar approach to the transmission line insertion, but instead of modifying its length, we have varied the characteristic impedance of the transmission line itself. The benefit of this solution are simplicity, enhanced performance and lower cost.

\section{THEORY}

A good background on the theory that supports our approach can be found somewhere else, ${ }^{5,6}$ but here we have summarized the relevant facts behind our proposal.

\section{Optimal Transmission Line Length}

Figure 2 shows a loss free transmission line terminated in an arbitrary load impedance $\mathrm{Z}_{\mathrm{L}}$.

If the incident and reflecte waves have the form
RECEIVED 11/12/98; ACCEPTED 4/18/99.

Address correspondence to Palmira Villa, Unidad de RMN. UCM, Paseo Juan XXIII, 1. Madrid 28040, Spain. Tel.: 34-91-
394-3272; Fax: 34-91-394-3245; E-mail: palmira@sgirmn. pluri.ucm.e 
A

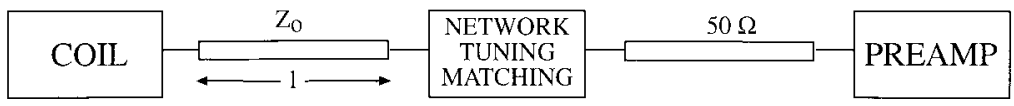

B

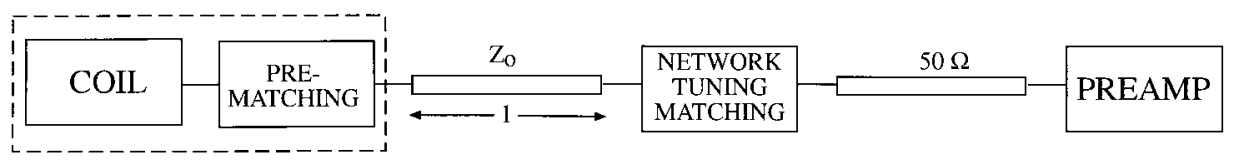

Fig. 1. A. Block diagram of a conventional remotely tuned transmission line $(l)$ circuit. B. Block diagram of a partially matched, remotely tuned transmission line $(l)$ circuit.

$V_{0}^{+} e^{j \beta z}$ and $V_{0}^{-} e^{j \beta z}$ respectively, the total voltage for a travelling wave on the line can be written as the sum of both waves:

$$
V(Z)=V_{0}^{+} e^{-j \beta z}+V_{0}^{-} e^{j \beta z}
$$

where $\beta$ (phase constant) is equal to $\frac{2 \pi}{\lambda}, \lambda$ being the wavelength.

Assuming a loss free transmission line, the input impedance seen from the electronics end, $Z_{I N}$, depends on its length, $l$, according to the following expression:

$$
Z_{I N}=Z_{0} \frac{\left(Z_{L}+j Z_{0} \tan \beta l\right)}{\left(Z_{0}+j Z_{L} \tan \beta l\right)}
$$

where $Z_{0}$ is the characteristic impedance of the transmission line. Depending on this length $Z_{I N}$ takes different values, and this is the matching technique proposed by Cross et al. ${ }^{1}$ and Gordon et al. ${ }^{2}$ For example, when $l=$ $\lambda / 4$ (or $\lambda / 4+\mathrm{n} \lambda / 2$ ), the input impedance is $Z_{I N}=\frac{Z_{0}^{2}}{Z_{L}}$, and for $l=\lambda / 2$ (or $\mathrm{n} \lambda / 2)$, the input impedance is $Z_{I N}=$ $Z_{L}$. In both cases, the imaginary part is zero. According to this, a half-wavelength line (or any multiple of $\lambda / 2$ ) does not transform the load impedance, regardless of the

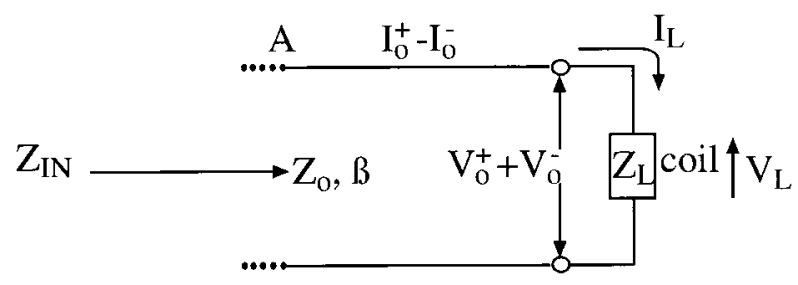

Fig. 2. Terminated transmission line. characteristic impedance. For lines with $\lambda / 4<l<\lambda / 2$, the input impedance is

$$
Z_{I N}=Z_{0} \frac{2 Z_{L} Z_{0}+j\left(Z_{L}^{2}-Z_{0}^{2}\right.}{Z_{0}^{2}+Z_{L}^{2}}
$$

For lines with $\lambda / 2<l<3 \lambda / 4$, the input impedance is

$$
Z_{I N}=Z_{0} \frac{2 Z_{L} Z_{0}+j\left(Z_{0}^{2}-Z_{L}^{2}\right)}{Z_{0}^{2}+Z_{L}^{2}}
$$

where in both cases the imaginary part is $\neq 0$.

The choice of an optimal $l$ is then a trade-off between the minimum transmission line length, which allows for the remote tuning and matching (and which minimizes the losses with a real transmission line) and a convenient length that cancels the $Z_{I N}$ imaginary part (phase equals zero on the reflectio coefficient $l=\lambda / 4$ for $n=1$ ).

\section{Efficienc}

The mismatch between the transmission line and the probe causes a reflectio at this junction, and subsequently an increased power dissipation in the line itself, reducing the efficienc in relation to the locally tuned case. The amount of reflectio can be quantifie with the reflectio coefficien $\rho$, define as

$$
\rho=\frac{Z_{L}-Z_{0}}{Z_{L}+Z_{0}}
$$

In a perfect matching between cable and coil, $\rho$ is equal to zero $0, Z_{0}$ being the characteristic impedance of the transmission line equal to the load impedance $Z_{L}$; in this case, the transmitted power is 


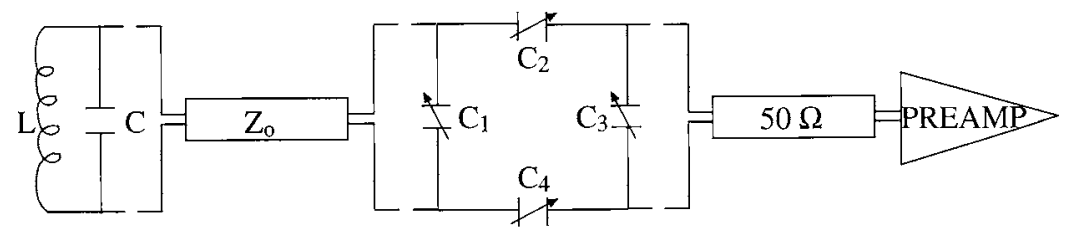

Fig. 3. Diagram of our remotely tuned scheme with a balanced capacitive network tuning-matching.

$$
P=\frac{1}{2} \frac{\left(V_{0}^{+}\right)^{2}}{Z_{0}}
$$

When there is a mismatch at the load $\left(Z_{L} \neq Z_{0}\right)$ the total power delivered to $Z_{L}$ is equal to the incident power minus the reflecte power:

$$
P=\frac{1}{2} \frac{\left(V_{0}^{+}\right)^{2}}{Z_{0}}\left(1-\rho^{2}\right)
$$

Substantial improvement maybe obtained with only partial tuning and matching of the probe. ${ }^{4}$ By placing fixe local capacitors at the detector coil in the conventional parallel-series configuration the imaginary part of the impedance can be cancelled and its module can be increased close to $50 \Omega$ at the junction with the coil. ${ }^{7}$

As an alternative, our proposal relies on the partial tuning of the probe by placing a local capacitor and, on the modificatio of the transmission line characteristic impedance, $Z_{0}$, to be as close as possible to the resulting probe impedance (Fig. 3).

\section{MATERIALS AND METHODS}

We have performed all the experiments with a Bruker Biospec 47/40 (proton resonant frequency $=200.36$ $\mathrm{MHz}$ ) spectrometer. Although any probe type is suitable for these tests, a well proben custom made surface coil (70 $\mathrm{mm}$ diameter) was used for all the experiments. The transmission lines considered were three cables with different characteristic impedances (Belden 9222, $50 \Omega$, used for local tuning; RG223, $50 \Omega$; and standard tv antenna parallel cable, $300 \Omega$ ).

We have compared the results of the remote matching scheme depicted in Fig. 3 with conventional local tuning. Two different transmission lines with different lengths were used: $l=\lambda / 2: 49 \mathrm{~cm}$ for the RG223, $1.41 \mathrm{~dB} / 10 \mathrm{~m}$ attenuation at $100 \mathrm{MHz}$, and $52 \mathrm{~cm}$ for the parallel cable, $1.68 \mathrm{~dB} / 10 \mathrm{~m}$ attenuation at $1 \mathrm{GHz}$. The distance $\lambda / 2$ was chosen instead of the minimum $\lambda / 4$ since the last one was not sufficien to allow mechanically accessible remote tuning and matching in the narrow magnet gantry. In this case there are two close resonant frequencies besides resonance $f_{0}\left(f_{o} / 2\right.$ and $\left.3 f_{o} / 2\right)$, but they are far enough apart to be considered. Longer lines such as $l=3 \lambda / 4$ will have two frequencies under $f_{0}$ and two frequencies above, frequencies that will be closer to the resonant working frequency, and then better filter located between the probe and the preamplifie are needed to accurately remove these frequencies.

The efficienc of these schemes was evaluated by measuring the amplifie attenuation for two different $90^{\circ}$ pulses $(2000 \mu \mathrm{s}$ and $3500 \mu$ s lengths $)$ when loaded with a spherical phantom (67 $\mathrm{mm}$ diameter) fille with doped water (copper sulfate). The radio frequency amplifie attenuation was registered instead of the pulse amplitude itself, since the former is a parameter directly provided by the system. Consequently, less attenuation means less efficiency

Signal-to-noise (SNR) measurements were computed from spin echo images (TR/TE: 2000:15 ms). Eight consecutive axial slices with $2 \mathrm{~mm}$ slice thickness were obtained in all cases using a fiel of view (FOV) of 80 $\mathrm{mm}$ and a $256 \times 256$ acquisition matrix. Only the central image was used for the calculations. Both signal and noise were measured over a circular region of interest (ROI) of $10 \times 10$ pixel size. For the evaluation of the signal six ROIs were selected in the region of highest homogeneity to minimize the impact of the surface coil sensitivity profil on the data. For the noise estimation, an ROI was placed in the corners of the FOV, on the image background. SNR was computed in two different ways. A) $\mathrm{SNR}_{1}$ is a ratio of the average of the six (one for each signal ROI) mean signal values $\mathrm{x}_{\mathrm{S}}$ and the average of the four (one for each noise ROI) $\sigma_{\mathrm{r}}, S N R_{1}=\frac{\bar{x}_{s}}{\sigma_{r}}$. B) $\mathrm{SNR}_{2}$ was computed according to the Henkelman equation, ${ }^{8}$ which introduced correction factors to obtain the amplitude signal in the presence of

Table 1. Pulse attenuation $(\mathrm{dB})$ and efficienc

\begin{tabular}{lccc}
\hline $\begin{array}{c}\text { Pulse length } \\
(\mu \mathrm{s})\end{array}$ & Conventional & $\begin{array}{c}\text { RG223 } \\
(50 \Omega)\end{array}$ & $\begin{array}{c}\text { Parallel } \\
(300 \Omega)\end{array}$ \\
\hline 2000 & 16.0 & 15.0 & 15.9 \\
3500 & 19.7 & 17.7 & 19.3 \\
Efficienc & & $71 \%$ & $94 \%$ \\
\hline
\end{tabular}


Table 2. Signal-to-noise-ratio and SWR

\begin{tabular}{|c|c|c|c|c|c|c|}
\hline \multirow[b]{2}{*}{ Pulse length $(\mu \mathrm{s})$} & \multicolumn{2}{|c|}{ Conventional (local) } & \multicolumn{2}{|c|}{ 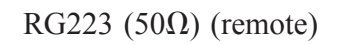 } & \multicolumn{2}{|c|}{ Parallel (300 $\Omega$ ) (remote) } \\
\hline & $\mathrm{SNR}_{2}$ & $\mathrm{SNR}_{1}$ & $\mathrm{SNR}_{2}$ & $\mathrm{SNR}_{1}$ & $\mathrm{SNR}_{2}$ & $\mathrm{SNR}_{1}$ \\
\hline $2000 \mu \mathrm{s}$ & 108.74 & 165.85 & 84.39 & 128.71 & 90.37 & 137.89 \\
\hline $3500 \mu \mathrm{s}$ & 125.46 & 192.52 & 89.13 & 135.94 & 91.07 & 138.99 \\
\hline SWR & & & \multicolumn{2}{|c|}{3.33} & \multicolumn{2}{|c|}{1.65} \\
\hline
\end{tabular}

SWR, standing wave ratio.

$S N R_{1}=\frac{\overline{x_{s}}}{\sigma_{r}}$ and $\mathrm{SNR}_{2}$ was computed according to Henkelman 8

noise, $S N R_{2}=\frac{A}{\sigma}, \sigma=\frac{\sigma_{\mathrm{r}}}{0.655}, \mathrm{~A}=\sqrt{\overline{\mathrm{x}}_{\mathrm{s}}^{2}+\sigma_{s}^{2}-2 \sigma^{2}}$ where $\sigma_{\mathrm{r}}$ is the noise standard deviation, $\mathrm{x}_{\mathrm{s}}$ the signal mean value, and $\sigma_{\mathrm{s}}$ the signal standard deviation.

\section{RESULTS AND DISCUSSION}

Pulse attenuation in $\mathrm{dB}$ and efficienc for all the studied cases are presented in Table 1. Probe efficienc increases as the characteristic impedance of the transmission line increases. Although we have only limited our analysis to two types of cables ( $50 \Omega$ and $300 \Omega$ ), it is expected that better results could be obtained if a higher impedance transmission line were used. ${ }^{7}$ Accordingly, and since this is not a comparison of probe performances rather a tuning and matching characterization, this technique is suitable for use with other kinds of probes or coils, as well as with other nuclei.

One of the disadvantages of the remote tuning and matching scheme is the narrow frequency range for the tuning condition of the probe $\left(5 \%\right.$ of $\left.\mathrm{f}_{0}\right) .{ }^{4}$ In the present case, this range mainly depends on the $\mathrm{C}_{1}$ capacitor in Fig. 3, and with a $30 \mathrm{pF}$ capacitor we have been able to obtain a $10 \%$ margin of the pre-tuned $\mathrm{f}_{0}$ for the matching condition. Although this wide margin may look excessive, there are circumstances were it may be necessary, such as, for example those cases where the size of the coil or its geometry limits the proper distribution of capacitors (miniature probes), or when the load is unpredictable and can vary in a wide range (implantable/intraoperative probes).

The efficienc improvement obtained with the proposed scheme has been evaluated and demonstrated as a function of two complementary and practical criteria: the SNR of acquired spin-echo images and the standing wave ratio (SWR) (Table 2). As can be observed from this table, SNRs increase with the impedance value. These magnitudes in both remote circuits tested depend on the pulse length, and are close to the ones obtained with local matching and tuning when shorter pulses are used.
The calculated SWR for our scheme using a parallel cable is within the optimal range (1.5-2). As is well known, a reduction in the reflectio coefficien signifi cantly decreases the losses. ${ }^{4}$ In our examples, lower losses introduced by the higher impedance transmission line inserted have shown significan effect on the evaluated parameters. However, there is a range in which further decreases in SWR values do not translate into critical deterioration of image quality.

In conclusion, we have presented an efficien technique for remote tuning and matching of probes, that in practice results in a substantial reduction of the experiment set up time in certain in vivo applications in which access to the probe is difficult

Acknowledgments-We acknowledge the Spanish Comision Interministerial de Ciencia y Tecnologia (CICYT) for funding (BIO97-0543).

\section{REFERENCES}

1. Cross, V.R.; Hester, R.K.; Waugh, J.S. Single coil probe with transmission line tuning for nuclear magnetic double resonance. Rev. Sci. Instrum. 47:1486-1487; 1976.

2. Gordon, R.E.; Timms, W.E. An improved tune and match circuit for Bo shimming in intact biological samples. J. Magn. Reson. 46:322-324; 1982.

3. Martin, J.F.; Daly, C.P. Transmission line matching of surface coils. Magn. Reson. Med. 3:346-351; 1986.

4. Rath, A.R. Efficien remote transmission line probe tuning. Magn. Reson. Med. 13:370-377; 1990.

5. Collins, R.E. Foundations for Microwave Engineering, $2^{\text {nd }}$ edn. New York: McGraw-Hill; 1992.

6. Pozar, D.M. Microwave Engineering. New York: Addison-Wesley; 1990.

7. Chen, C.; Hoult, D.I. Biomedical Magnetic Resonance Technology. Bristol, England: Adam Hilber; 1989.

8. Henkelman, R.M. Measurement of signal intensities in the presence of noise in MR images. Med. Phys. 12:232-3; 1985.

9. Hoult, D.I.; Richard, R.E. The signal to noise ratio of nuclear magnetic resonance experiment. J. Magn. Reson. 24:71-85; 1976. 